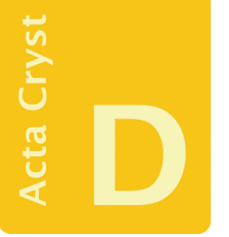

\title{
STRUCTURAL
} BIOLOGY

Volume 76 (2020)

Supporting information for article:

Attaining atomic resolution from in situ data collection at room temperature using counterdiffusion-based low-cost microchips

Jose A. Gavira, Isaac Rodriguez-Ruiz, Sergio Martinez-Rodriguez, Shibom Basu, Sébastien Teychené, Andrew A. McCarthy and Christoph Mueller-Dieckman 


\section{Glucose Isomerase}
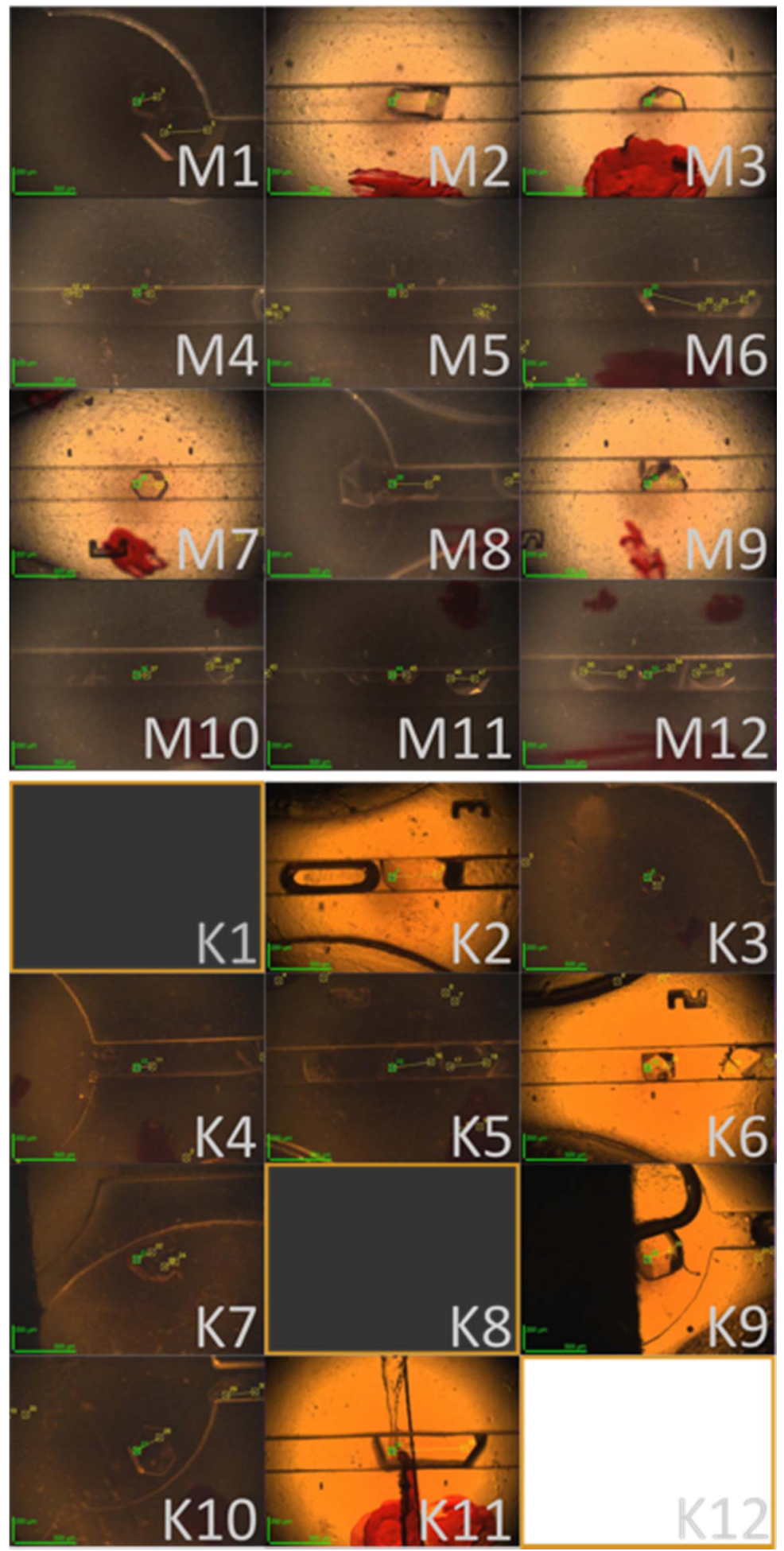

Figure S1 Snapshot pictures of the counter-diffusion crystallization experiments of glucose isomerase implemented in Mylar (M1 to M12) and Kapton (K1to K12) microchips. Green-path on the crystals shows initial and final points of the helical trajectories followed for data collection. 


\section{Lysozyme}
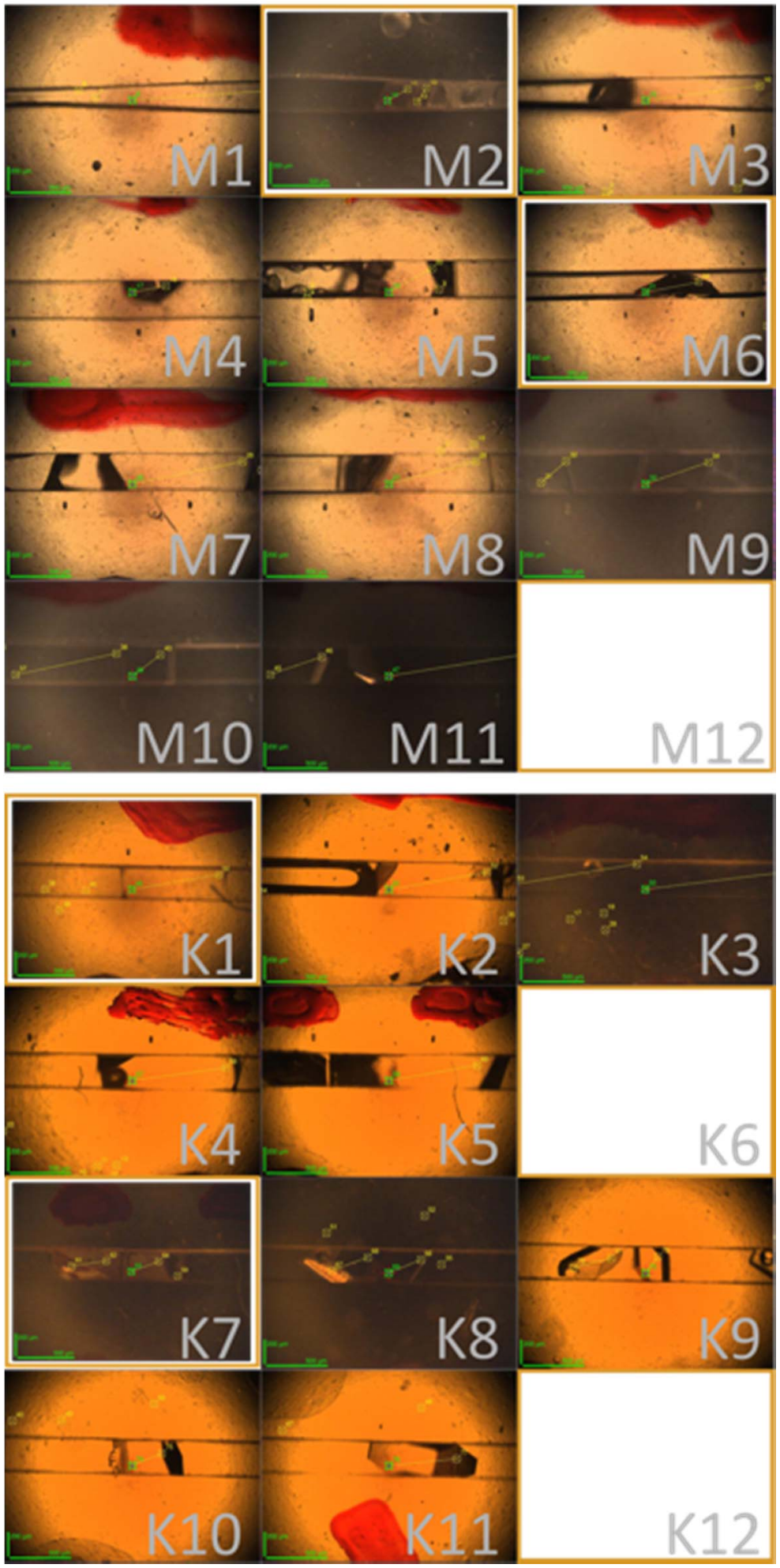

Figure S2 Snapshot pictures of the counter-diffusion crystallization experiments of lysozyme implemented in Mylar (M1 to M12) and Kapton (K1to K12) microchips. Green-path on the crystals shows initial and final points of the helical trajectories followed for data collection. 


\section{Thaumatin}
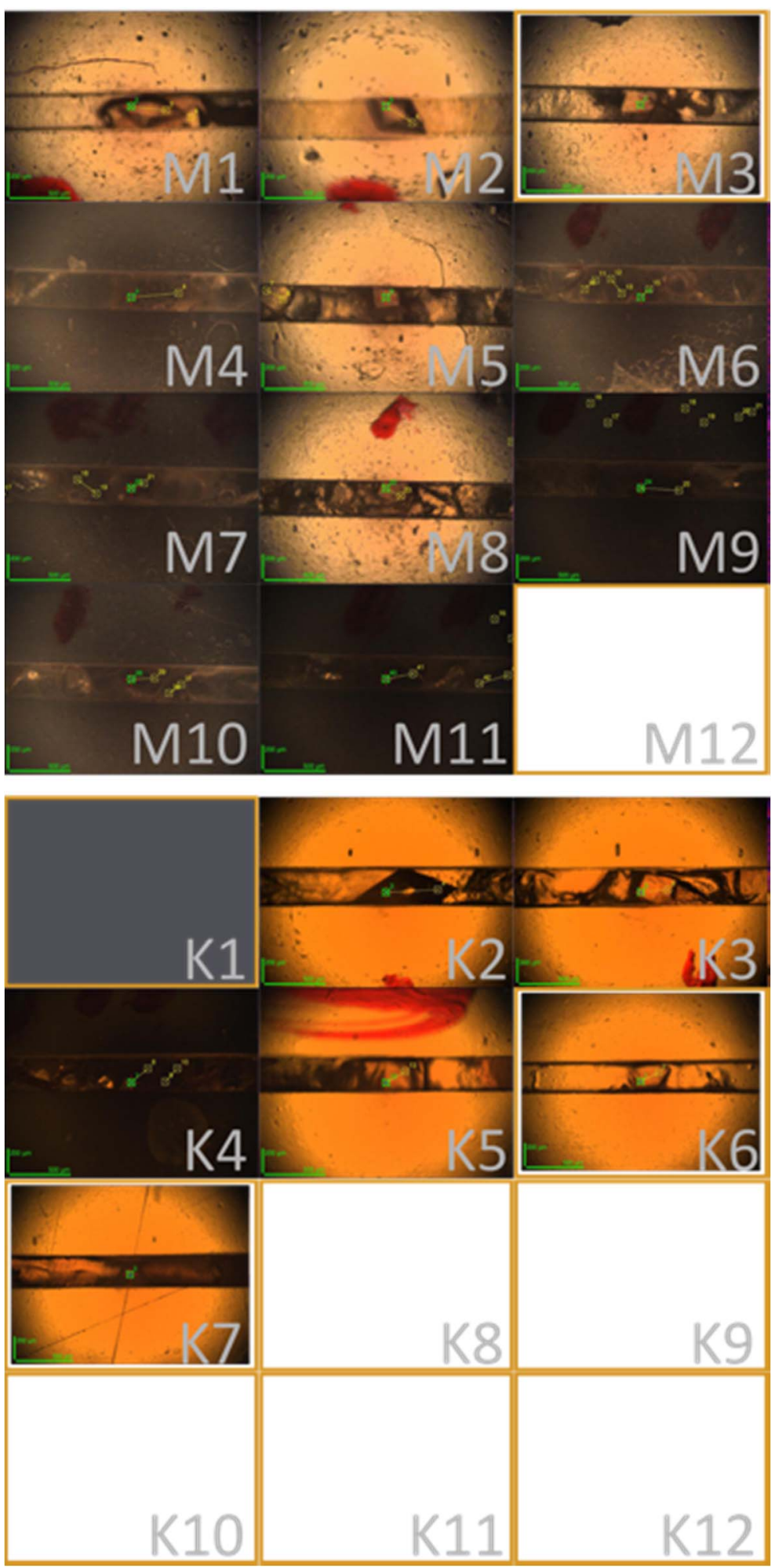

Figure S3 Snapshot pictures of the counter-diffusion crystallization experiments of thaumatin implemented in Mylar (M1 to M12) and Kapton (K1 to K12) microchips. Green-path on the crystals shows initial and final points of the helical trajectories followed for data collection. 


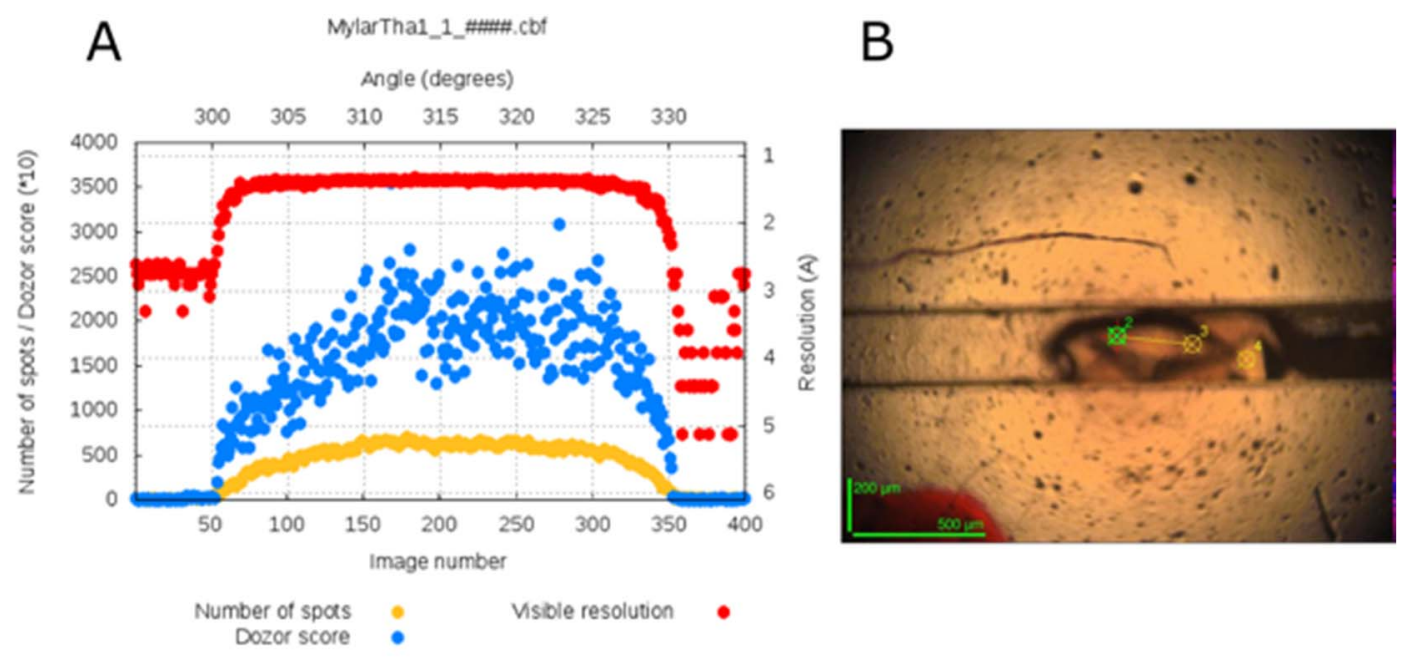

Figure S4 A. Snapshot pictures of the Dozor score output of the data collection of a thaumatin crystal exemplifying the difficulties in properly centering the crystals in the plane perpendicular to the X-ray beam. B. Helical trajectory (2 to 3 ) followed during data collection. 


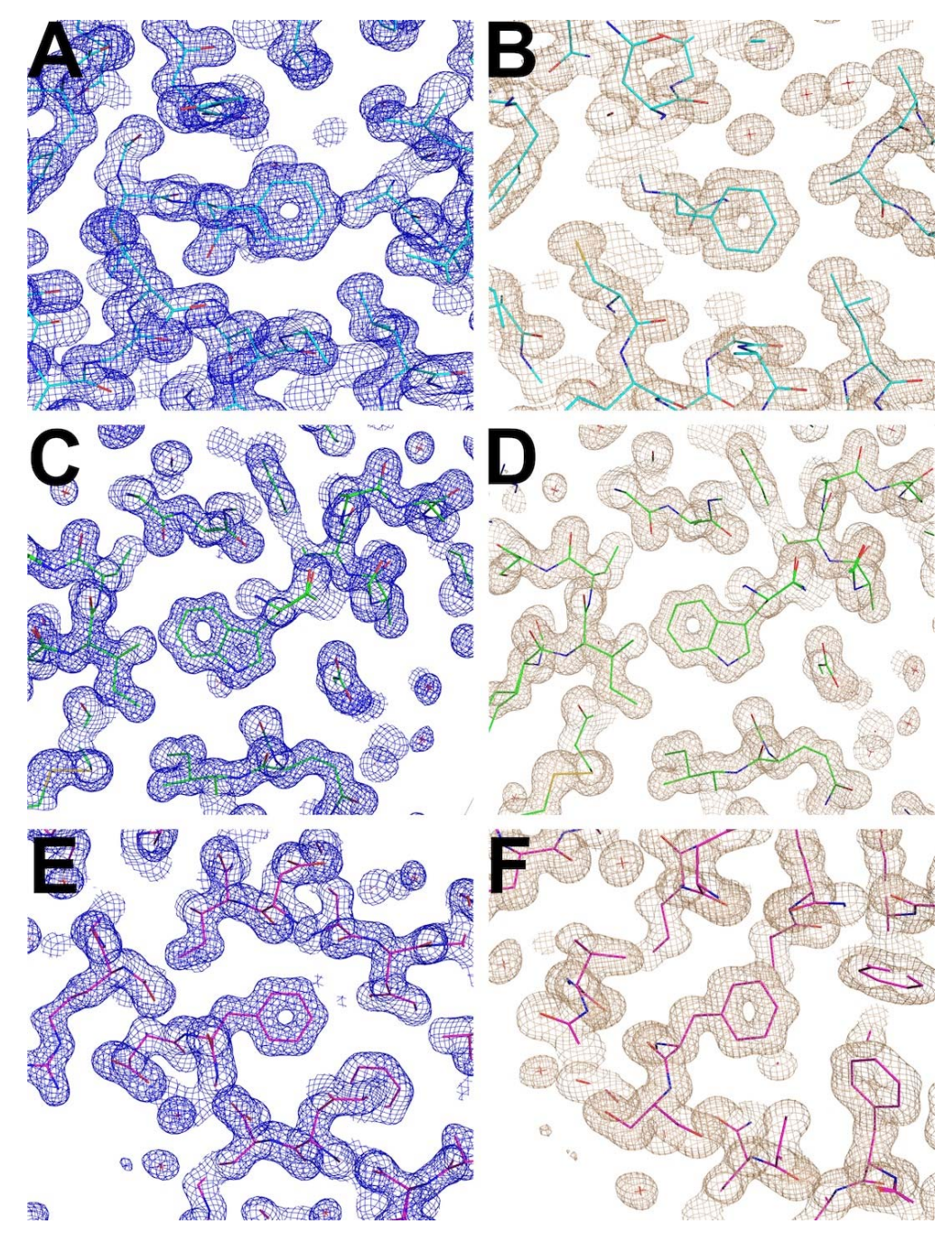

Figure S5 Snapshot of the $|2 \mathrm{Fo}-\mathrm{Fc}|$ electron-density maps contoured at $1.0 \sigma$ showing the built models of glucose isomerase (cyan), lysozyme (green) and thaumatin (pink). A, C, E (blue) and B, D, E (brownish) maps correspond to crystals grown in Mylar and Kapton chips, respectively.

\section{Supplementary multimedia}

Supplementary Movie S1 represents a full dataset collected by rotating $45^{\circ}$. 
Table S1 Summary of the data collection conditions and final statistical values for glucose isomerase crystals grown in the Kapton microchips

The final statistical values are from XSDAPP, XIA2, EDNA or aimless (note as ccp4).

\begin{tabular}{|c|c|c|c|c|c|c|c|c|c|}
\hline Dataset & KGlu2 & KGlu3 & KGlu4 & KGlu5 & KGlu6 & KGlu7 & KGlu9 & KGlu10 & KGlu11 \\
\hline \multicolumn{10}{|l|}{ Data Acquisition } \\
\hline ESRF Beam-line & ID30B & ID30B & ID30B & ID30B & ID30B & ID30B & ID30B & ID30B & ID30B \\
\hline Detector type & Pilatus3 6M & Pilatus3 6M & Pilatus3 6M & Pilatus3 6M & Pilatus3 6M & Pilatus3 6M & Pilatus3 6M & Pilatus3 6M & Pilatus3 6M \\
\hline Wavelength ( $)$ & 0.9763 & 0.9763 & 0.9763 & 0.9763 & 0.9763 & 0.9763 & 0.9763 & 0.9763 & 0.9763 \\
\hline Distance (mm) & 268 & 188 & 188 & 188 & 188 & 188 & 159 & 159 & 159 \\
\hline Number of images & 400 & 400 & 450 & 450 & 450 & 450 & 450 & 450 & 450 \\
\hline Oscillation $\left(^{\circ}\right)$ & 0.1 & 0.1 & 0.1 & 0.1 & 0.1 & 0.1 & 0.1 & 0.1 & 0.1 \\
\hline Transmission (\%) & 9.8 & 9.8 & 9.8 & 9.8 & 9.8 & 9.8 & 19.2 & 9.8 & 9.8 \\
\hline Exposure time (ms) & 0.02 & 0.02 & 0.02 & 0.02 & 0.02 & 0.02 & 0.02 & 0.02 & 0.02 \\
\hline Data Statistics & XDSAPP & XIA2 & XIA2 & EDNA/XIA2 & autoPROC & EDNA/ccp4 & EDNA/ccp4 & XIA2 & XIA2 \\
\hline Space group & I 222 & I 222 & I 222 & I 222 & I 222 & I 222 & I 222 & I 222 & I 222 \\
\hline $\begin{array}{l}\text { Unit cell } \\
\qquad \mathrm{a}, \mathrm{b}, \mathrm{c}(\AA)\end{array}$ & $\begin{array}{c}93.95,99.36 \\
103.05\end{array}$ & $\begin{array}{c}94.03,99.37 \\
103.07\end{array}$ & $\begin{array}{c}93.95,99.37 \\
103.06\end{array}$ & $\begin{array}{c}94.03,99.41 \\
103.09\end{array}$ & $\begin{array}{c}94.05,99.40 \\
103.06\end{array}$ & $\begin{array}{c}94.06,99.42 \\
103.24\end{array}$ & $\begin{array}{c}93.97,99.42 \\
103.05\end{array}$ & $\begin{array}{c}94.03,99.35 \\
103.07\end{array}$ & $\begin{array}{c}93.99,99.36 \\
103.05\end{array}$ \\
\hline Resolution ( $\AA$ ) & $41.13-1.50$ & $40.01-1.23$ & $40.00-1.08$ & $41.15-1.12$ & $51.53-1.35$ & $41.19-1.30$ & $41.13-1.15$ & $39.29-1.16$ & $41.13-1.11$ \\
\hline (High shell) & $(1.40-1.50)$ & $(1.25-1.23)$ & $(1.10-1.08)$ & $(1.15-1.12)$ & $(1.37-1.35)$ & $(1.33-1.30)$ & $(1.17-1.15)$ & $(1.19-1.16)$ & $(1.14-1-11)$ \\
\hline Unique reflections & 50656 & 91046 & 124576 & 142169 & 55424 & 101639 & 98050 & 141313 & 157647 \\
\hline R-merge $*(\%)$ & $8.7(9.6)$ & $7.9(51.8)$ & $7.5(49.0)$ & $8.2(35.6)$ & $9.3(38.5)$ & $6.2(65.9)$ & $5.1(18.4)$ & $6.4(55.0)$ & $10.5(53.1)$ \\
\hline$<\mathrm{I} / \sigma(\mathrm{I})>$ & $9.2(7.5)$ & $5.0(1.1)$ & $5.4(1.0)$ & $5.0(1.1)$ & $5.6(2.1)$ & $5.2(0.9)$ & $14.8(2.3)$ & $5.4(1.3)$ & $3.7(0.9)$ \\
\hline Completeness (\%) & $68.3(70.8)$ & $65.8(66.9)$ & $61.3(35.1)$ & $77.7(65.1)$ & $52.1(56.7)$ & $86.5(90.5)$ & $57.9(47.4)$ & $85.9(81.7)$ & $84.0(84.1)$ \\
\hline Multiplicity & $1.4(1.4)$ & $2.1(2.1)$ & $2.3(1.6)$ & $1.9(1.4)$ & $2.2(2.0)$ & $1.9(1.8)$ & $1.9(1.4)$ & $1.8(1.8)$ & $1.9(1.8)$ \\
\hline $\mathrm{CC}(1 / 2)$ & $98(97)$ & $98(62)$ & $98.9(66.1)$ & $97.7(61.5)$ & $98.6(84.5)$ & $97.5(61.5)$ & $99.2(95.3)$ & $98.4(55.8)$ & $96.1(62.7)$ \\
\hline B-factor $\left(\AA^{2}\right)$ & 11.7 & 13.7 & 13.5 & 12.6 & 14.68 & 13.8 & 12.5 & 11.6 & 11.8 \\
\hline Mosaicity $\left({ }^{\circ}\right)$ & 0.016 & 0.04 & 0.11 & 0.08 & 0.2 & 0.08 & 0.04 & 0.03 & 0.01 \\
\hline
\end{tabular}




\begin{tabular}{lccccc}
\hline Dataset & KGlu3b & KGlu4b & KGlu5b & KGlu7b & KGlu10-2 \\
\hline Data Acquisition & & & & & \\
ESRF Beam-line & ID30B & ID30B & ID30B & ID30B & ID30B \\
Detector type & Pilatus3 6M & Pilatus3 6M & Pilatus3 6M & Pilatus3 6M & Pilatus3 6M \\
Wavelength $(\AA)$ & 0.9763 & 0.9763 & 0.9763 & 0.9763 & 0.9763 \\
Distance (mm) & 188 & 188 & 188 & 188 & 159 \\
Number of images & 400 & 450 & 450 & 450 & 450 \\
Oscillation ( $\left.{ }^{\circ}\right)$ & 0.1 & 0.1 & 0.1 & 0.1 & 0.1 \\
Transmission $(\%)$ & 9.8 & 9.8 & 9.8 & 9.8 & 9.8 \\
Exposure time $(\mathrm{ms})$ & 0.02 & 0.02 & 0.02 & 0.02 & 0.02 \\
\hline Data Statistics & XIA2 & XIA2 & EDNA/ccp4 & EDNA/ccp4 & EDNA/ccp4 \\
\hline Space group & I 222 & I 222 & I 222 & I 222 & I 222 \\
Unit cell & $94.04,99.36$, & $94.012,99.33$, & $93.99,99.38$, & $94.01,99.40$, & $94.03,99.35$ \\
a, b, c $(\AA)$ & 103.07 & 103.045 & 103.07 & 103.10 & 103.07 \\
Resolution $(\AA)$ & $47.02-1.28$ & $41.13-1.20$ & $41.14-1.05$ & $41.14-1.67$ & $39.29-1.16$ \\
(High shell) & $(1.31-1.28)$ & $(1.22-1.20)$ & $(1.07-1.05)$ & $(1.70-1.67)$ & $(1.17-1.15)$ \\
Unique reflections & 81005 & 111428 & 138864 & 43838 & 119599 \\
R-merge * $(\%)$ & $6.3(67.4)$ & $7.4(52.5)$ & $5.8(59.4)$ & $5.1(6.6)$ & $5.9(47.5)$ \\
$<\mathrm{I} / \sigma(\mathrm{I})>$ & $6.1(1.0)$ & $5.8(1.0)$ & $5.9(1.2)$ & $17.0(10.8)$ & $4.8(0.7)$ \\
Completeness $(\%)$ & $66.0(69.0)$ & $74.4(81.20)$ & $62.6(30.1)$ & $78.8(75.3)$ & $70.7(61.8)$ \\
Multiplicity & $2.2(1.9)$ & $2.1(1.9)$ & $1.8(1.0)$ & $1.9(1.7)$ & $1.7(1.5)$ \\
CC(1/2) & $98.6(55.4)$ & $98.1(59.1)$ & $99.1(89.7)$ & $99.2(98.1)$ & $99.0(84.6)$ \\
B-factor $\left(\AA^{2}\right)$ & 13.57 & 13.47 & 11.6 & 12.7 & 12.8 \\
Mosaicity $\left({ }^{\circ}\right)$ & 0.046 & 0.067 & 0.03 & 0.06 & 0.03 \\
\hline
\end{tabular}


Table S2 Summary of the data collection conditions and final statistical values for glucose isomerase crystals grown in the Mylar microchips.

The final statistical values are from XSDAPP, XIA2, EDNA or aimless (note as ccp4).

\begin{tabular}{|c|c|c|c|c|c|c|c|c|c|}
\hline Dataset & MylarGlu1 & MylarGlu2 & MylarGlu3 & MylarGlu4 & MylarGlu5 & MylarGlu6 & MylarGlu7 & MylarGlu8 & MylarGlu9 \\
\hline \multicolumn{10}{|l|}{ Data Acquisition } \\
\hline ESRF Beam-line & ID30B & ID30B & ID30B & ID30B & ID30B & ID30B & ID30B & ID30B & ID30B \\
\hline Detector type & Pilatus3 6M & Pilatus3 6M & Pilatus3 6M & Pilatus3 6M & Pilatus3 6M & Pilatus3 6M & Pilatus3 6M & Pilatus3 6M & Pilatus3 6M \\
\hline Wavelength $(\AA)$ & 0.9763 & 0.9763 & 0.9763 & 0.9763 & 0.9763 & 0.9763 & 0.9763 & 0.9763 & 0.9763 \\
\hline Distance (mm) & 188 & 188 & 188 & 215 & 215 & 188 & 188 & 188 & 188 \\
\hline Number of images & 450 & 450 & 450 & 450 & 450 & 450 & 450 & 450 & 450 \\
\hline Oscillation $\left(^{\circ}\right)$ & 0.1 & 0.1 & 0.1 & 0.1 & 0.1 & 0.1 & 0.1 & 0.1 & 0.1 \\
\hline Transmission $(\%)$ & 9.8 & 9.8 & 9.8 & 9.8 & 9.8 & 9.8 & 9.8 & 9.8 & 9.8 \\
\hline Exposure time (ms) & 0.02 & 0.02 & 0.02 & 0.02 & 0.02 & 0.02 & 0.02 & 0.02 & 0.02 \\
\hline Data Statistics & EDNA/ccp4 & EDNA & parallelProc & XIA2 & EDNA & autoProc/ccp4 & EDNA/ccp4 & EDNA/ccp4 & autoProc \\
\hline Space group & I 222 & I 222 & I 222 & I 222 & I 222 & I 222 & I 222 & I 222 & I 222 \\
\hline $\begin{array}{l}\text { Unit cell } \\
\qquad \mathrm{a}, \mathrm{b}, \mathrm{c}(\AA)\end{array}$ & $\begin{array}{c}93.99,99.37 \\
103.10\end{array}$ & $\begin{array}{c}95.93,99.40 \\
104.87\end{array}$ & $\begin{array}{c}94.01,99.40 \\
103.10\end{array}$ & $\begin{array}{c}94.016,99.42 \\
103.09\end{array}$ & $\begin{array}{c}94.08,99.40 \\
103.10\end{array}$ & $\begin{array}{c}93.94,99.38 \\
103.10\end{array}$ & $\begin{array}{c}93.93,99.32 \\
103.01\end{array}$ & $\begin{array}{c}93.98,99.35 \\
103.01\end{array}$ & $\begin{array}{c}93.93,99.41, \\
103.07\end{array}$ \\
\hline Resolution $(\AA)$ & $39.28-1.10$ & $42.13-1.18$ & $39.29-1.30$ & $68.30-1.26$ & $47.04-1.30$ & 41.14-1.06 & $47.01-1.20$ & $40.30-1.05$ & $41.13-1.15$ \\
\hline (High shell) & $(1.12-1.10)$ & $(1.22-1.18)$ & $(1.32-1.30)$ & $(1.29-1.26)$ & $(1.39-1.30)$ & $(1.10-1.06)$ & $(1.22-1.20)$ & $(1.07-1.15)$ & $(1.17-1.15)$ \\
\hline Unique reflections & 123280 & 112849 & 100012 & 109177 & 90258 & 152288 & 111745 & 151745 & 133170 \\
\hline R-merge $*(\%)$ & $5.6(25.9)$ & $12.1(47.0)$ & $7.6(61.1)$ & $6.5(59.3)$ & $4.3(77.7)$ & $7.7(42.5)$ & $5.6(66.2)$ & $5.8(64.5)$ & $6.8(28.3)$ \\
\hline$<\mathrm{I} / \sigma(\mathrm{I})>$ & $10.7(3.0)$ & $3.3(0.9)$ & $3.7(1.0)$ & 5.5. (1.1) & $8.4(1.1)$ & $7.3(1.2)$ & $4.1(1.1)$ & $12.5(1.2)$ & $6.8(2.2)$ \\
\hline Completeness $(\%)$ & $64.0(33.6)$ & $65.9(54.5)$ & $85.2(88.1)$ & $84.8(87.5)$ & $76.9-79.9$ & $70.9(31.9)$ & $75.2(69.0)$ & $68.3(25.8)$ & $71.8(70.9)$ \\
\hline Multiplicity & $1.8(1.5)$ & $2.2(2.0)$ & $1.8(1.8)$ & $1.9(1.8)$ & $2.1(2.0)$ & $1.9(1.5)$ & $1.9(1.8)$ & $1.7(1.2)$ & $1.8(1.6)$ \\
\hline $\mathrm{CC}(1 / 2)$ & $99.0(91.6)$ & $96.4(62.9)$ & $98.4(67.0)$ & $97.9(48.0)$ & $99.7(48.5)$ & $98.3(64.9)$ & $99.2(77.5)$ & $99.0(75.0)$ & $98.9(88.9)$ \\
\hline B-factor $\left(\AA^{2}\right)$ & 11.4 & 9.7 & 12.9 & 23.78 & 13.7 & 18.3 & 12.8 & 12.0 & 13.2 \\
\hline Mosaicity $\left({ }^{\circ}\right)$ & 0.03 & 0.03 & 0.00 & 0.08 & 0.03 & 0.02 & 0.02 & 0.03 & 0.06 \\
\hline
\end{tabular}




\begin{tabular}{|c|c|c|c|c|c|}
\hline Dataset & MylarGlu6b & MylarGlu8b & MylarGlu10 & MylarGlu11 & MylarGlu12 \\
\hline \multicolumn{6}{|l|}{ Data Acquisition } \\
\hline ESRF Beam-line & ID30B & ID30B & ID30B & ID30B & ID30B \\
\hline Detector type & Pilatus3 6M & Pilatus3 6M & Pilatus3 6M & Pilatus3 6M & Pilatus3 6M \\
\hline Wavelength $(\AA)$ & 0.9763 & 0.9763 & 0.9763 & 0.9763 & 0.9763 \\
\hline Distance (mm) & 188 & 188 & 188 & 242 & 242 \\
\hline Number of images & 450 & 450 & 450 & 450 & 450 \\
\hline Oscillation $\left({ }^{\circ}\right)$ & 0.1 & 0.1 & 0.1 & 0.1 & 0.1 \\
\hline Transmission (\%) & 9.8 & 9.8 & 9.8 & 9.8 & 9.8 \\
\hline Exposure time (ms) & 0.02 & 0.02 & 0.02 & 0.02 & 0.02 \\
\hline Data Statistics & autoPROC/ccp4 & EDNA/ccp4 & EDNA/ccp4 & autoProc & EDNA/ccp4 \\
\hline Space group & I 222 & I 222 & I 222 & I 222 & I 222 \\
\hline $\begin{array}{l}\text { Unit cell } \\
\text { a, b, c ( }(\AA)\end{array}$ & $\begin{array}{c}93.94,99.38 \\
103.10\end{array}$ & $\begin{array}{c}93.93,99.32 \\
103.01\end{array}$ & $\begin{array}{c}93.96,99.39, \\
103.07\end{array}$ & $\begin{array}{c}94.03,99.38, \\
103.07\end{array}$ & $\begin{array}{c}93.94,99.41, \\
103.09\end{array}$ \\
\hline Resolution $(\AA)$ & 41.14-1.06 & $40.39-1.10$ & $39.27-1.25$ & $69.46-1.15$ & $40.42-1.18$ \\
\hline (High shell) & $(1.10-1.06)$ & $(1.12-1.10)$ & $(1.27-1.25)$ & $(1.17-1.15)$ & $(1.21-1.18)$ \\
\hline Unique reflections & 152288 & 129386 & 90696 & 103887 & 116638 \\
\hline R-merge $*(\%)$ & $7.7(42.5)$ & $4.6(74.3)$ & $9.1(59.9)$ & $4.9(29.4)$ & $8.2(30.9)$ \\
\hline$<\mathrm{I} / \sigma(\mathrm{I})>$ & $7.3(1.2)$ & $11.2(1.4)$ & $7.1(1.2)$ & $10.2(2.0)$ & $6.0(1.0)$ \\
\hline Completeness (\%) & $70.9(31.9)$ & $67.0(40.9)$ & $68.9(69.4)$ & $61.8(14.8)$ & $74.5(23.4)$ \\
\hline Multiplicity & $1.9(1.5)$ & $2.0(1.5)$ & $2.3(2.2)$ & $1.7(1.1)$ & $1.7(1.3)$ \\
\hline $\mathrm{CC}(1 / 2)$ & $98.3(64.9)$ & $99.4(79.0)$ & $98.7(61.6)$ & $99.4(87.0)$ & $96.9(86.6)$ \\
\hline B-factor $\left(\AA^{2}\right)$ & 18.3 & 12.3 & 13.1 & 15.18 & 13.1 \\
\hline Mosaicity $\left({ }^{\circ}\right)$ & 0.02 & 0.03 & 0.03 & 0.03 & 0.02 \\
\hline
\end{tabular}


Table S3 Summary of the data collection conditions and final statistical values for lysozyme crystals grown in the Mylar microchips.

The final statistical values are from XSDAPP, XIA2, EDNA or aimless (note as ccp4).

\begin{tabular}{|c|c|c|c|c|c|c|c|c|c|}
\hline Dataset & MylarLzm1 & MylarLzm3 & MylarLzm4 & MylarLzm5 & MylarLzm7 & MylarLzm8 & MylarLzm9 & MylarLzm10 & MylarLzm11 \\
\hline \multicolumn{10}{|l|}{ Data Acquisition } \\
\hline ESRF Beam-line & ID30B & ID30B & ID30B & ID30B & ID30B & ID30B & ID30B & ID30B & ID30B \\
\hline Detector type & Pilatus3 6M & Pilatus3 6M & Pilatus3 6M & Pilatus3 6M & Pilatus3 6M & Pilatus3 6M & Pilatus3 6M & Pilatus3 6M & Pilatus3 6M \\
\hline Wavelength $(\AA)$ & 0.9763 & 0.9763 & 0.9763 & 0.9763 & 0.9763 & 0.9763 & 0.9763 & 0.9763 & 0.9763 \\
\hline Distance $(\mathrm{mm})$ & 242 & 242 & 242 & 242 & 242 & 242 & 242 & 242 & 242 \\
\hline Number of images & 400 & 400 & 400 & 400 & 450 & 450 & 450 & 450 & 450 \\
\hline Oscillation $\left(^{\circ}\right)$ & 0.1 & 0.1 & 0.1 & 0.1 & 0.1 & 0.1 & 0.1 & 0.1 & 0.1 \\
\hline Transmission $(\%)$ & 100 & 9.8 & 9.8 & 9.8 & 9.8 & 9.8 & 9.8 & 9.8 & 9.8 \\
\hline Exposure time (ms) & 0.02 & 0.02 & 0.02 & 0.02 & 0.02 & 0.02 & 0.02 & 0.02 & 0.02 \\
\hline Data Statistics & XDSAPP/ccp4 & autoProc/ccp4 & XDSAPP/ccp4 & parallel & XDSAPP/ccp4 & EDNA/ccp4 & XDSAPP & EDNA & autoProc/ccp4 \\
\hline Space group & $\mathrm{P} 4{ }_{3} 2{ }_{1} 2$ & $\mathrm{P} 4{ }_{3} 2_{1} 2$ & $\mathrm{P} 43_{3} 2$ & $\mathrm{P} 42_{3} 2$ & $\mathrm{P} 4{ }_{3} 2_{1} 2$ & $\mathrm{P} 4{ }_{3} 2_{1} 2$ & $\mathrm{P} 4_{3} 2_{1} 2$ & $\mathrm{P} 4_{3} 2_{1} 2$ & $\mathrm{P} 43_{3} 2$ \\
\hline \multicolumn{10}{|l|}{ Unit cell } \\
\hline $\mathrm{a}=\mathrm{b}, \mathrm{c}(\AA)$ & $78.48,37.53$ & $79.15,37.97$ & $78.85,38.30$ & $78.82,38.27$ & $79.08,37.86$ & $79.18,37.93$ & $79.19,37.97$ & $79.17,37.97$ & $79.18,37.97$ \\
\hline Resolution $(\AA)$ & $39.24-1.85$ & $34.23-1.20$ & $35.50-1.31$ & $39.40-1.12$ & $39.54-1.27$ & $27.99-1.19$ & $34.24-1.17$ & $37.97-1.12$ & $37.97-1.14$ \\
\hline (High shell) & $(1.89-1.85)$ & $(1.22-1.20)$ & $(1.33-1.31)$ & $(1.16-1.12)$ & $(1.31-1.27)$ & $(1.21-1.19)$ & $(1.19-1.17)$ & $(1.16-1.12)$ & $(1.16-1.14)$ \\
\hline Unique reflections & 5729 & 19568 & 23567 & 20612 & 29621 & 30310 & 31586 & 32304 & 32079 \\
\hline R-merge $*(\%)$ & $30.0(72.5)$ & $6.9(50.8)$ & $8.0(47.6)$ & $6.5(45.0)$ & $7.0(30.7)$ & $5.5(31.4)$ & $9.2(28.7)$ & $6.0(32.5)$ & $8.2(33.1)$ \\
\hline$<\mathrm{I} / \sigma(\mathrm{I})>$ & $4.8(2.5)$ & $11.5(2.0)$ & $10.1(2.2)$ & $15.9(1.7)$ & $8.1(1.7)$ & $12.8(1.1)$ & $8.7(2.2)$ & $13.1(1.4)$ & $10.4(2.2)$ \\
\hline Completeness (\%) & $54.1(59.5)$ & $52.6(21.1)$ & $80.6(74.1)$ & $44.0(6.3)$ & $92.8(82.9)$ & $78.0(42.6)$ & $77.1(35.0)$ & $70.3(15.8)$ & $72.3(17.8)$ \\
\hline Multiplicity & $4.7(4.6)$ & $4.3(3.3)$ & $3.2(2.4)$ & $4.0(1.7)$ & $3.1(2.0)$ & $3.2(1.6)$ & $3.0(1.6)$ & $3.1(1.1)$ & $2.9(1.2)$ \\
\hline $\mathrm{CC}(1 / 2)$ & $94.1(62.0)$ & $99.1(61.5)$ & $99.1(72.8)$ & $99.4(77.4)$ & $99.1(82.9)$ & $99.3(82.5)$ & $98.9(86.8)$ & $99.4(74.0)$ & $98.6(84.1)$ \\
\hline B-factor $\left(\AA^{2}\right)$ & 13.9 & 15.5 & 14.1 & 15.1 & 17.18 & 14.7 & 16.7 & 14.6 & 15.6 \\
\hline Mosaicity $\left(^{\circ}\right)$ & 0.311 & 0.31 & 0.02 & 0.03 & 0.06 & 0.04 & 0.03 & 0.03 & 0.02 \\
\hline
\end{tabular}




\begin{tabular}{|c|c|c|c|c|c|}
\hline Dataset & MylarLzm9b & MylarLzm10b & MylarLzm10b & MylarLzm11b & MylarLzm11c \\
\hline \multicolumn{6}{|l|}{ Data Acquisition } \\
\hline ESRF Beam-line & ID30B & ID30B & ID30B & ID30B & ID30B \\
\hline Detector type & Pilatus3 6M & Pilatus3 6M & Pilatus3 6M & Pilatus3 6M & Pilatus3 6M \\
\hline Wavelength $(\AA)$ & 0.9763 & 0.9763 & 0.9763 & 0.9763 & 0.9763 \\
\hline Distance (mm) & 242 & 242 & 242 & 242 & 242 \\
\hline Number of images & 450 & 450 & 450 & 450 & 450 \\
\hline Oscillation $\left({ }^{\circ}\right)$ & 0.1 & 0.1 & 0.1 & 0.1 & 0.1 \\
\hline Transmission (\%) & 9.8 & 9.8 & 9.8 & 9.8 & 9.8 \\
\hline Exposure time (ms) & 0.02 & 0.02 & 0.02 & 0.02 & 0.02 \\
\hline Data Statistics & XDSAPP & EDNA & EDNA & autoProc/ccp4 & autoProc/ccp4 \\
\hline Space group & $\mathrm{P} 4_{3} 2_{1} 2$ & $\mathrm{P} 44_{3} 2_{1} 2$ & $\mathrm{P} 44_{3} 2_{1}$ & $\mathrm{P} 42_{3} 2$ & $\mathrm{P} 4_{3} 2_{12}$ \\
\hline $\begin{array}{l}\text { Unit cell } \\
\qquad \mathrm{a}=\mathrm{b}, \mathrm{c}(\AA)\end{array}$ & $79.19,37.97$ & $79.18,37.97$ & $79.18,37.97$ & $79.18,37.97$ & $79.11,38.02$ \\
\hline $\begin{array}{c}\text { Resolution }(\AA) \\
\text { (High shell) }\end{array}$ & $\begin{array}{l}34.24-1.15 \\
(1.17-1.15)\end{array}$ & $\begin{array}{c}37.97-1.12 \\
(1.16-1.12)\end{array}$ & $\begin{array}{c}34.25-1.16 \\
(1.18-1.16)\end{array}$ & $\begin{array}{c}34.24-1.14 \\
(1.17-1.14)\end{array}$ & $\begin{array}{c}37.97-1.12 \\
(1.14-1.12)\end{array}$ \\
\hline Unique reflections & 32346 & 32009 & 31774 & 31061 & 30859 \\
\hline R-merge $*(\%)$ & $8.5(43.1)$ & $6.4(28.4)$ & $8.2(32.8)$ & $6.8(31.7)$ & $6.4(23.2)$ \\
\hline$<\mathrm{I} / \sigma(\mathrm{I})>$ & $9.4(2.0)$ & $12.9(1.6)$ & $9.0(0.9)$ & $10.0(0.9)$ & $8.9(2.0)$ \\
\hline Completeness (\%) & $75.8(28.8)$ & $69.5(15.1)$ & $75.1(30.5)$ & $70.9(19.1)$ & $67.4(8.7)$ \\
\hline Multiplicity & $2.9(1.1)$ & $3.0(1.1)$ & $3.1(1.2)$ & $3.2(1.3)$ & $3.1(1.1)$ \\
\hline $\mathrm{CC}(1 / 2)$ & $98.8(81.5)$ & $99.2(69.3)$ & $98.6(72.4)$ & $98.8(75.6)$ & $99.1(82.0)$ \\
\hline B-factor $\left(\AA^{2}\right)$ & 16.2 & 14.6 & 14.9 & 14.8 & 15.3 \\
\hline Mosaicity $\left({ }^{\circ}\right)$ & 002 & 0.03 & 0.08 & 0.02 & 0.02 \\
\hline
\end{tabular}


Table S4 Summary of the data collection conditions and final statistical values for lysozyme crystals grown in the Kapton microchips.

The final statistical values are from XSDAPP, XIA2, EDNA or aimless (note as ccp4).

\begin{tabular}{|c|c|c|c|c|c|c|c|c|c|}
\hline Dataset & K-Lzm2 & K-Lzm3 & K-Lzm4 & K-Lzm5 & K-Lzm8 & K-Lzm8 & K-Lzm9 & K-Lzm10 & K-Lzm11 \\
\hline \multicolumn{10}{|l|}{ Data Acquisition } \\
\hline ESRF Beam-line & ID30B & ID30B & ID30B & ID30B & ID30B & ID30B & ID30B & ID30B & ID30B \\
\hline Detector type & Pilatus3 6M & Pilatus3 6M & Pilatus3 6M & Pilatus3 6M & Pilatus3 6M & Pilatus3 6M & Pilatus3 6M & Pilatus3 6M & Pilatus3 6M \\
\hline Wavelength ( $\AA$ ) & 0.9763 & 0.9763 & 0.9763 & 0.9763 & 0.9763 & 0.9763 & 0.9763 & 0.9763 & 0.9763 \\
\hline Distance (mm) & 242 & 242 & 188 & 188 & 188 & 188 & 188 & 188 & 188 \\
\hline Number of images & 450 & 450 & 470 & 500 & 500 & 500 & 450 & 450 & 450 \\
\hline Oscillation $\left(^{\circ}\right)$ & 0.1 & 0.1 & 0.1 & 0.1 & 0.1 & 0.1 & 0.1 & 0.1 & 0.1 \\
\hline Transmission (\%) & 9.8 & 9.8 & 9.8 & 19.2 & 9.8 & 9.8 & 9.8 & 9.8 & 9.8 \\
\hline Exposure time (ms) & 0.02 & 0.02 & 0.02 & 0.02 & 0.02 & 0.02 & 0.02 & 0.02 & 0.02 \\
\hline Data Statistics & EDNA & XIA2 & EDNA/ccp4 & EDNA/ccp4 & EDNA/ccp4 & autoProc/ccp4 & parallel & EDNA/ccp4 & EDNA/ccp4 \\
\hline Space group & $\mathrm{P} 4{ }_{3} 2_{1} 2$ & $\mathrm{P} 4_{3} 2_{1} 2$ & $\mathrm{P} 4{ }_{3} 2_{1} 2$ & $\mathrm{P} 4{ }_{3} 2_{1} 2$ & $\mathrm{P} 4{ }_{3} 2_{1} 2$ & $\mathrm{P} 4{ }_{3} 2{ }_{1} 2$ & $\mathrm{P} 4{ }_{3} 2_{1} 2$ & $\mathrm{P} 4{ }_{3} 2_{1} 2$ & $\mathrm{P} 4{ }_{3} 2_{1} 2$ \\
\hline \multicolumn{10}{|l|}{ Unit cell } \\
\hline $\mathrm{a}=\mathrm{b}, \mathrm{c}(\AA)$ & $79.14,37.97$ & $79.14,38.99$ & $78.91,38.01$ & $78.88,37.96$ & $79.11,38.02$ & $79.11,38.03$ & $79.14,38.03$ & $79.11,38.03$ & $79.11,38.05$ \\
\hline Resolution ( $\AA$ ) & $39.57-1.19$ & $38.00-1.20$ & $39.46-1.10$ & $39.44-1.55$ & $34.27-1.10$ & $55.94-1.13$ & $38.03-1.14$ & $35.28-1.15$ & $34.29-1.15$ \\
\hline (High shell) & $(1.23-1.19)$ & $(1.23-1.20)$ & $(1.12-1.10)$ & $(1.58-1.55)$ & $(1.12-1.10)$ & $(1.15-1.13)$ & $(1.18-1.14)$ & $(1.17-1.15)$ & $(1.17-1.15)$ \\
\hline Unique reflections & 34031 & 33642 & 46282 & 12437 & 45994 & 40888 & 39675 & 35695 & 33808 \\
\hline R-merge $*(\%)$ & $7.0(50.8)$ & $8.3(29.4)$ & $6.5(82.8)$ & $14.5(89.5)$ & $6.5(87.1)$ & $9.7(40.0)$ & $8.5(75.4)$ & $7.4(66.2)$ & $8.8(65.0)$ \\
\hline$<\mathrm{I} / \sigma(\mathrm{I})>$ & $8.0(1.3)$ & $10.1(1.2)$ & $8.4(0.8)$ & $8.0(3.6)$ & $11.5(1.1)$ & $7.5(2.2)$ & $7.9(1.1)$ & $13.0(1.1)$ & $10.6(1.3)$ \\
\hline Completeness (\%) & $87.0(49.6)$ & $58.5(59.0)$ & $94.7(68.2)$ & $69.5(74.1)$ & $93.7(68.7)$ & $90.4(70.6)$ & $90.3(81.6)$ & $82.8(84.7)$ & $79.2(71.5)$ \\
\hline Multiplicity & $2.9(1.5)$ & $2.0(1.1)$ & $3.1(2.2)$ & $4.5(4.4)$ & $3.2(2.0)$ & $3.1(2.6)$ & $3.1(2.5)$ & $3.4(2.6)$ & $3.8(3.2)$ \\
\hline $\mathrm{CC}(1 / 2)$ & $99.1(75.5)$ & 98.0 (79.9) & $99.1(45.5)$ & $96.6(39.8)$ & $99.3(68.7)$ & $98.8(84.5)$ & $98.6(53.4)$ & $99.1(63.6)$ & $99.1(68.2)$ \\
\hline B-factor $\left(\AA^{2}\right)$ & 15.4 & 14.13 & 14.1 & 13.4 & 14.6 & 16.2 & 14.3 & 14.9 & 14.4 \\
\hline Mosaicity $\left({ }^{\circ}\right)$ & 0.05 & 0.00 & 0.05 & 0.28 & 0.03 & 0.02 & 0.03 & 0.03 & 0.03 \\
\hline
\end{tabular}




\begin{tabular}{lc}
\hline Dataset & K-Lzm9b \\
\hline Data Acquisition & \\
ESRF Beam-line & ID30B \\
Detector type & Pilatus3 6M \\
Wavelength $(\AA)$ & 0.9763 \\
Distance $(\mathrm{mm})$ & 188 \\
Number of images & 450 \\
Oscillation $\left({ }^{\circ}\right)$ & 0.1 \\
Transmission $(\%)$ & 9.8 \\
Exposure time $(\mathrm{ms})$ & 0.02 \\
\hline Data Statistics & parallel \\
\hline Space group & $\mathrm{P} 43212$ \\
Unit cell & \\
a $=$ b, c $(\AA)$ & $79.14,38.03$ \\
Resolution $(\AA)$ & $34.27-1.18$ \\
(High shell) & $(1.20-1.18)$ \\
Unique reflections & 34498 \\
R-merge $*(\%)$ & $9.4(85.4)$ \\
$<\mathrm{I} / \sigma(\mathrm{I})>$ & $6.9(1.4)$ \\
Completeness $(\%)$ & $86.6(84.9)$ \\
Multiplicity & $3.5(3.4)$ \\
CC $(1 / 2)$ & $98.6(5394)$ \\
B-factor $\left(\AA^{2}\right)$ & 15.6 \\
Mosaicity $\left({ }^{\circ}\right)$ & 0.01 \\
\hline &
\end{tabular}


Table S5 Summary of the data collection conditions and final statistical values for thaumatin crystals grown in the Kapton microchips. The final statistical values are from XSDAPP, XIA2, EDNA or aimless (note as ccp4).

\begin{tabular}{|c|c|c|c|c|}
\hline Dataset & K-Thau2 & K-Thau3 & K-Thau4 & K-Thau5 \\
\hline \multicolumn{5}{|l|}{ Data Acquisition } \\
\hline ESRF Beam-line & ID30B & ID30B & ID30B & ID30B \\
\hline Detector type & Pilatus3 6M & Pilatus3 6M & Pilatus3 6M & Pilatus3 6M \\
\hline Wavelength $(\AA)$ & 0.9763 & 0.9763 & 0.9763 & 0.9763 \\
\hline Distance (mm) & 317 & 187 & 267 & 267 \\
\hline Number of images & 400 & 400 & 450 & 450 \\
\hline Oscillation $\left(^{\circ}\right)$ & 0.1 & 0.1 & 0.1 & 0.1 \\
\hline Exposure time (ms) & 0.02 & 0.02 & 0.02 & 0.02 \\
\hline Transmission $(\%)$ & 9.8 & 9.8 & 9.8 & 9.8 \\
\hline Data Statistics & autoPro/ccp4 & autoPro/ccp4 & XIA2 & EDNA/ccp4 \\
\hline Space group & $\mathrm{P} 4_{1} 2_{1} 2$ & $\mathrm{P} 4_{1} 2_{1} 2$ & $\mathrm{P} 4_{1} 22_{1}$ & $\mathrm{P} 4_{1} 2_{12}$ \\
\hline \multicolumn{5}{|l|}{ Unit cell } \\
\hline $\mathrm{a}, \mathrm{b}, \mathrm{c}(\AA)$ & $58.28,151.45$ & $58.50,151.44$ & $58.19,151.35$ & $57.18,149.04$ \\
\hline Resolution $(\AA)$ & 39.76-1.32 & $54.57-1.30$ & $54.31-1.43$ & $45.37-2.10$ \\
\hline (High shell) & $(1.34-1.32)$ & $(1.32-1.30)$ & $(1.45-1.43)$ & $(2.16-2.10)$ \\
\hline Unique reflections & 40601 & 45819 & 46125 & 9496 \\
\hline $\mathrm{R}$-merge $*(\%)$ & $4.1(25.7)$ & $6.1(45.2)$ & $4.6(62.7)$ & $7.8(68.0)$ \\
\hline$<\mathrm{I} / \sigma(\mathrm{I})>$ & $10.7(1.6)$ & $7.4(1.5)$ & $9.8(1.0)$ & $6.9(0.9)$ \\
\hline Completeness $(\%)$ & $65.8(10.9)$ & $71.0(57.8)$ & $94.0(89.9)$ & $62.6(50.1)$ \\
\hline Multiplicity & $2.5(1.0)$ & $3.1(1.4)$ & $2.8(2.4)$ & $2.6(2.4)$ \\
\hline $\mathrm{CC}(1 / 2)$ & 99.7 (84.6) & $99.4(81.4)$ & $98.8(63.6)$ & $99.6(62.8)$ \\
\hline B-factor $\left(\AA^{2}\right)$ & 16.7 & 17.5 & 18.51 & 31.7 \\
\hline Mosaicity $\left(^{\circ}\right)$ & 0.04 & 0.03 & 0.01 & 0.86 \\
\hline
\end{tabular}


Table S6 Summary of the data collection conditions and final statistical values for lysozyme crystals grown in the Mylar microchips.

The final statistical values are from XSDAPP, XIA2, EDNA or aimless (note as ccp4).

\begin{tabular}{|c|c|c|c|c|c|c|c|c|c|}
\hline Dataset & MylarThau1 & MylarThau2 & MylarThau4 & MylarThau4b & MylarThau5 & MylarThau6 & MylarThau7 & MylarThau8 & MylarThau9 \\
\hline \multicolumn{10}{|l|}{ Data Acquisition } \\
\hline Detector type & Pilatus3 6M & Pilatus3 6M & Pilatus3 6M & Pilatus3 6M & Pilatus3 6M & Pilatus3 6M & Pilatus3 6M & Pilatus3 6M & Pilatus3 6M \\
\hline Distance (mm) & 267 & 267 & 267 & 267 & 267 & 267 & 188 & 188 & 268 \\
\hline Number of images & $400 / 300$ & $400 / 250$ & 400 & 400 & 400 & 400 & 400 & 400 & 400 \\
\hline Oscillation $\left({ }^{\circ}\right)$ & 0.1 & 0.1 & 0.1 & 0.1 & 0.1 & 0.1 & 0.1 & 0.1 & 0.1 \\
\hline Transmission (\%) & 100 & 100 & 100 & 9.8 & 9.8 & 9.8 & 19.2 & 19.2 & 19.2 \\
\hline Space group & P $4{ }_{1} 2_{1} 2$ & P $4{ }_{1} 2_{1} 2$ & $\mathrm{P} 4_{1} 2_{1} 2$ & $\mathrm{P} 44_{1} 22_{2}$ & $\mathrm{P} 4_{1} 2_{1} 2$ & $\mathrm{P} 4_{1} 2_{1} 2$ & $\mathrm{P} 44_{1} 22_{2}$ & $\mathrm{P} 4_{1} 2_{1} 2$ & $\mathrm{P} 4_{1} 2_{12}$ \\
\hline $\begin{array}{l}\text { Unit cell } \\
\text { a, b, c ( }(\AA)\end{array}$ & $58.58,151.54$ & $58.61,151.58$ & $58.55,151.58$ & $58.52,151.55$ & $58.49,151.71$ & $58.57,151.56$ & $58.57,151.51$ & $58.57,151.55$ & $58.57,151.57$ \\
\hline $\begin{array}{c}\text { Resolution }(\AA) \\
\text { (High shell) }\end{array}$ & $\begin{array}{l}38.26-1.40 \\
(1.42-1.40)\end{array}$ & $\begin{array}{l}39.98-1.30 \\
(1.32-1.30)\end{array}$ & $\begin{array}{l}46.34-1.20 \\
(1.22-1.20)\end{array}$ & $\begin{array}{l}54.59-1.35 \\
(1.39-1.35)\end{array}$ & $\begin{array}{l}41.36-1.38 \\
(1.40-1.38)\end{array}$ & $\begin{array}{l}41.41-1.40 \\
(1.42-1.40)\end{array}$ & $\begin{array}{l}54.63-1.30 \\
(1.33-1.30)\end{array}$ & $\begin{array}{l}46.34-1.35 \\
(1.37-1.35)\end{array}$ & $\begin{array}{l}46.34-1.25 \\
(1.27-1.25)\end{array}$ \\
\hline Unique reflections & 45224 & 48703 & 55591 & 54511 & 37150 & 48286 & 57029 & 55886 & 57570 \\
\hline $\mathrm{CC}(1 / 2)$ & $98.5(47.5)$ & $96.5(45.5)$ & $99.4(95.9)$ & $98.4(71.1)$ & $99.2(57.9)$ & $98.8(59.9)$ & $98.2(52.3)$ & $98.3(53.4)$ & $98.6(90.8)$ \\
\hline B-factor $\left(\AA^{2}\right)$ & 16.9 & 12.9 & 13.8 & 14.1 & 16.4 & 15.1 & 14.2 & 13.1 & 13.8 \\
\hline Mosaicity $\left({ }^{\circ}\right)$ & 0.13 & 0.09 & 0.04 & 0.01 & 0.01 & 0.02 & 0.02 & 0.03 & 0.03 \\
\hline
\end{tabular}




\begin{tabular}{|c|c|c|c|c|c|}
\hline Dataset & MylarThau9b & MylarThau10b & MylarThau11 & MylarThau13 & MylarThau14b \\
\hline \multicolumn{6}{|l|}{ Data Acquisition } \\
\hline ESRF Beam-line & ID30B & ID30B & ID30B & ID30B & ID30B \\
\hline Detector type & Pilatus3 6M & Pilatus3 6M & Pilatus3 6M & Pilatus3 6M & Pilatus3 6M \\
\hline Wavelength $(\AA)$ & 0.9763 & 0.9763 & 0.9763 & 0.9763 & 0.9763 \\
\hline Distance (mm) & 187 & 187 & 188 & 188 & 188 \\
\hline Number of images & 400 & 400 & 400 & 400 & 400 \\
\hline Oscillation $\left({ }^{\circ}\right)$ & 0.1 & 0.1 & 0.1 & 0.1 & 0.1 \\
\hline Transmission (\%) & 19.2 & 19.2 & 9.8 & 9.8 & 9.8 \\
\hline Exposure time (ms) & 0.02 & 0.02 & 0.02 & 0.02 & 0.02 \\
\hline Data Statistics & XIA2 & XIA2 & XIA2/ccp4 & XIA2/ccp4 & XIA2/ccp4 \\
\hline Space group & $\mathrm{P} 44_{1} 2$ & $\mathrm{P} 4{ }_{1} 2,2$ & $\mathrm{P} 4_{1} 2_{1} 2$ & $\mathrm{P} 4_{1} 2_{1} 2$ & $\mathrm{P} 4_{1} 2_{1} 2$ \\
\hline \multicolumn{6}{|l|}{ Unit cell } \\
\hline a, b, c ( $(\AA)$ & $58.58,151.55$ & $58.58,151.56$ & $58.57,151.55$ & $58.57,151.55$ & $58.57,151.55$ \\
\hline Resolution $(\AA)$ & $54.64-1.25$ & $39.95-1.32$ & $54.63-1.32$ & $54.63-1.38$ & $38.25-1.34$ \\
\hline (High shell) & $(1.28-1.25)$ & $(1.35-1.32)$ & $(1.34-1.32)$ & $(1.40-1.38)$ & $(1.36-1.34)$ \\
\hline Unique reflections & 72059 & 60512 & 51725 & 49938 & 53759 \\
\hline R-merge * $(\%)$ & $7.6(73.4)$ & $8.8(91.5)$ & $10.3(78.4)$ & $10.3(69.8)$ & $10.3(65.0)$ \\
\hline$<\mathrm{I} / \sigma(\mathrm{I})>$ & $5.6(1.0)$ & $5.2(1.1)$ & $5.4(1.1)$ & $5.0(1.3)$ & $4.8(1.1)$ \\
\hline Completeness (\%) & $97.9(95.9)$ & $96.9(95.6)$ & $83.4(89.2)$ & $91.8(90.0)$ & $90.0(90.8)$ \\
\hline Multiplicity & $2.8(2.7)$ & $2.9(3.0)$ & $3.3(3.1)$ & $3.0(3.1)$ & $3.0(3.1)$ \\
\hline $\mathrm{CC}(1 / 2)$ & $98.4(57.4)$ & $98.0(51.0)$ & $95.1(50.7)$ & $98.4(64.7)$ & $98.4(59.2)$ \\
\hline B-factor $\left(\AA^{2}\right)$ & 14.9 & 14.6 & 16.5 & 15.1 & 15.5 \\
\hline Mosaicity $\left({ }^{\circ}\right)$ & 0.01 & 0.01 & 0.01 & 0.01 & 0.01 \\
\hline
\end{tabular}


Table S7 Resolution limit of the three model proteins, lysozyme, thaumatin and glucose isomerase, obtained from microfluidic systems compared with the data obtained in this work.

\begin{tabular}{|c|c|c|c|c|}
\hline \multirow[t]{2}{*}{ Material } & \multicolumn{4}{|c|}{ Resolution limit ( $(\AA)$} \\
\hline & Lysozyme & Thaumatin & Glucose Isomerase & Publication \\
\hline Kapton & 1.13 & 1.35 & 1.06 & This work \\
\hline Mylar & 1.12 & 1.14 & 1.20 & This work \\
\hline \multirow{3}{*}{$\mathrm{COC}$} & 1.75 & 2.00 & & (Ng et al., 2008) \\
\hline & 1.5 & 1.65 & & (Dhouib et al., 2009) \\
\hline & 1.43 & 1.55 & & (Pinker et al., 2013) \\
\hline \multirow{2}{*}{ PDMS } & & 1.25 & & (Hansen et al., 2006) \\
\hline & & 2.80 & & (Dhouib et al., 2009) \\
\hline PMMA & & 1.85 & & (Dhouib et al., 2009) \\
\hline \multirow{2}{*}{$\begin{array}{l}\text { Droplet } \\
\text { system }\end{array}$} & 1.9 & & & $\left(\right.$ Gerdts et al., 2008) ${ }^{*}$ \\
\hline & & & 2.1 & (Heymann et al., 2014) \\
\hline Kapton & - & 1.89 & 1.75 & (Gicquel et al., 2018) \\
\hline
\end{tabular}

*Polycarbonate 